\title{
Analytical and Numerical Investigation of Non- Newtonian Droplet Breakup in Asymmetric T-junction
}

\author{
M. J. Kiani ${ }^{1}$ and A. Bedram ${ }^{2 \dagger}$ \\ ${ }^{1}$ School of Mechanical Engineering, Iran University of Science and Technology, Tehran, Iran \\ ${ }^{2}$ Faculty of Imam Ali, South Khorasan Branch, Technical and Vocational University (TVU), Tabas, Iran
}

†Corresponding Author Email: abedram@tvu.ac.ir

(Received March 14, 2021; accepted October 20,2021)

\begin{abstract}
In this paper, an analytical investigation and 3D numerical simulation are presented for the breakup of floating non-Newtonian droplets in a non-Newtonian fluid. The considered geometry is a T-junction with unequal-width branches that can generate droplets with un-equal size. There is a very good agreement between the analytical solution and numerical simulation results obtained in this research. Various quantities such as branches flow rate ratio, branches velocity ratio, droplet's length in each branch, the whole length of the droplet, vorticity and pressure have been investigated during the breakup process in this study. The results showed that the branches flow rate ratio and the branches velocity ratio were constant during the breakup process. It was also observed that the length of the droplet in each of the branches and the whole length of the droplet increased linearly during the breakup process. Also, the vorticity has its maximum at the breakup moment.
\end{abstract}

Keywords: non-Newtonian fluid; Asymmetric T-junction; Analytical solution; Numerical simulation; 3D, VOF.

\section{NOMENCLATURE}

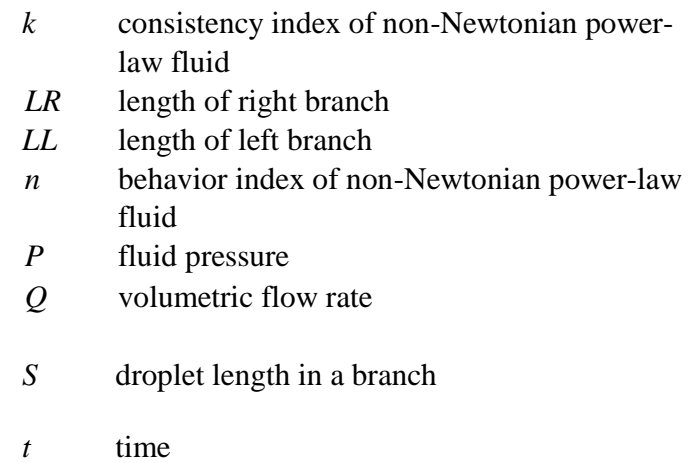

$\begin{array}{ll}U c & \text { continuous fluid velocity } \\ U d & \begin{array}{l}\text { droplet velocity } \\ \text { fluid velocity in a branch } \\ \text { channel width }\end{array} \\ \sigma & \begin{array}{l}\text { surface tension } \\ \text { dimensionless quantity of the horizontal } \\ \text { direction }\end{array} \\ \Gamma & \begin{array}{l}\text { dimensionless quantity of the vertical } \\ \text { direction }\end{array}\end{array}$

\section{INTRODUCTION}

Microfluidic processes are widely used in medical, Pharmaceutical, chemical, and other industries (Khoo et al. 2018; Xiong et al. 2018; Ruggeri et al. 2018). Among the microfluidic processes, dropletbased processes have particular importance (Maio and Dunlop 2018; Feng 2017; Mondal and Chatterjee 2016). In these processes, there is a base fluid into which secondary fluids are added in the shape of droplets. Advantages of this method include:

1. Droplets are not dissolved in the main fluid.

2. Droplets have no contact with the wall.

3. There is no droplet reaction with the wall

Droplet-based processes involve various operations, such as breakup (KianiMoqadam et al. 2018), generation, coalescence (Rahman et al. 2019), mixing (Ahmadi et al. 2019), and control (Yang et al. 2018).

In most droplet generation process, a T-junction is used to generate droplets (Liu et al. 2018). In this method, the secondary fluids are injected into the main fluid channel. As a result, secondary fluid 
droplets are formed in the main fluid. In the droplet generation process, there are also methods for generating a large number of droplets at a given time (Yadavali et al. 2018).

Various methods have been proposed for the breakup of droplets. In all these methods, an initial droplet is divided into two or more minor droplets. In some methods, a symmetric T-junction is used to breakup the droplet (Mora et al. 2018; Bedram and Moosavi 2013). These methods can generate only a specific size of the droplet (because they divide the droplet into two equal parts). A T-junction can separate two droplets close to each other (Deshpande and Dekker 2018). Using a T-junction with branches of unequal length, one can also divide a droplet or bubble into droplets of non-uniform size (Fu et al. 2014). The Tjunction with unequal-width branches can also divide an initial droplet into droplets of non-uniform size (Bedram and Moosavi 2011). By creating a wave, one can generate a droplet from the fluid of a channel (Hu and Cubaud 2018). By using a Tjunction with a valve, one can breakup an initial droplet into the droplets of the arbitrary size (Bedram et al. 2015a), there is also an analytical solution for this type of junction (Bedram et al. 2015b). Another method for generating unequal-sized bubbles is using a T-junction by which the initial bubble enters the system from one of the branches (Wang et al. 2014). After breakup, the smaller part enters the main channel of the T-junction, while the larger one enters the other branch. Wang et al. (2014) experimentally investigated this method, finding that the minimum width of bubble had linear variation with time. Also their research showed that the length of the initial bubble had no special effect on the breakup process.

In another way, by passing a bubble from a direct tube, the bubble is divided into smaller parts (Wang et al. 2017); the limitation of this method is that the generated droplets (after the breakup process) are located near each other; so we need another process for the separation of them.

When a droplet is generated in the T-junction, large vortexes are formed inside it; this significantly increases the mixing inside the droplet, making the material in it well mixed (Cui et al. 2020).

Amani et al. (2019) also studied the breakup of a bubble bypassing the bubble through a tube with varying cross-sections. Dai et al. (2017), on the other hand, examined the breakup of a bubble by passing the bubble through a hole. In their research, the bubble was floating in the non-Newtonian fluid. Further, Jejurkar et al. (2017) reviewed the breakup of the non-Newtonian fluid jet. However, the limitation of these three methods is that small and large droplets generated after the breakup are close together; therefore, another process is required for the separation of them.

Some researchers have focused on non-Newtonian droplets generation and droplet generation in nonNewtonian continuous fluid (Rostami and Morini 2020, Zhao et al. 2020). One of the common methods for the generation of non-Newtonian droplets is using a symmetric $\mathrm{T}$-junction in which the secondary fluid and the main fluid flow inside two perpendicular tubes. In this process, the secondary fluid is formed as droplets in the main fluid (Sontti and Atta 2017; Chiarello et al. 2017). In some studies, the generation of non-Newtonian droplets in a cross-junction has been investigated (Rostami and Morini 2018).

The droplet breakup process has numerous applications in the pharmaceutical and chemical industries (for the production and transfer of the nanoliter units of different materials). On the other hand, the use of non-Newtonian fluids is also common in those industries. Therefore, processes are needed to study the breakup of non-Newtonian droplets. One of the effective methods for the breakup of droplets is using an asymmetric $\mathrm{T}$ junction.

In the previous researches, the breakup of nonNewtonian droplets in a T-junction with unequal widths has not been studied; so we focused on this subject in this paper. Therefore, the novelty of this paper is the investigation of the non-Newtonian droplet breakup in a T-junction with unequal width branches. The geometry of this paper can produce unequal non-Newtonian droplets from an initial droplet. Also, the continuous fluid is considered nonNewtonian. The research method consists of the analytical solution and 3D numerical simulation using the VOF method. The analytical and numerical results of this study have a very good agreement. Also, the numerical results of this paper are in a close agreement with those of an analytical benchmark problem (Bretherton 1961). Also, grid independency and time step independency were checked for this study. By using the analytical solution of the present study, the flow rate ratio of branches, the velocity ratio of the fluid in the branches (for 3D geometry) and the droplet length during the breakup (for 2D geometry) were obtained. Also, the pressure distribution, velocity and vorticity inside the droplet at different moments during the breakup process were investigated. The results showed that the flow rate ratio of the branches and the velocity ratio of the branches were constant during the breakup process. It was also observed that the length of the droplet in each of the branches and the total length of the droplet increased linearly during the breakup process. The results also revealed that vorticity values in the regions near the droplet surface were 3 to 7 times higher than those in the middle of the droplet (therefore, the mixing of the materials inside the droplet increased). Also, the maximum vorticity (after the start of the droplet deforming process in the center of the junction until the end of the process) is related to the moment when the droplet is broken up and the two new droplets are separated.

\section{SYSTEM GEOMETRY}

The geometry of the problem (T-junction with unequal widths) is shown in Fig. 1. Non-Newtonian continuous fluid flow (wherein the droplets float) enters the vertical branch, leaving the two horizontal branches. The cross-section of all channels is rectangular. Depth of geometry (in the direction 
perpendicular to the paper) is $6 \mu \mathrm{m}$. The width of the right branch is $19 \mu \mathrm{m}$ and that of the left one is $20 \mu \mathrm{m}$. The input droplet length (the distance between points

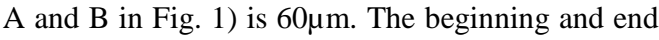
of the droplet are circles with a radius of $9 \mu \mathrm{m}$. The boundary condition of the input of the system is constant velocity, and the boundary condition at the outputs of the system (right and left branches) is constant pressure. The velocity fluid at the input of the system is $0.2 \mathrm{~m} / \mathrm{s}$. The continuous fluid is a nonNewtonian fluid with the consistency index $k=0.001$, a behavior index of $n=1.1$ and a density of $1000 \mathrm{~kg} / \mathrm{m}^{3}$. The droplet is a non-Newtonian fluid with the consistency index of $k=0.00125$, the behavior index of $n=1.1$ and the density of $800 \mathrm{~kg} / \mathrm{m}^{3}$. Surface tension between the two fluids is $0.005 \mathrm{~N} / \mathrm{m}$, with the contact angle of $180^{\circ}$.

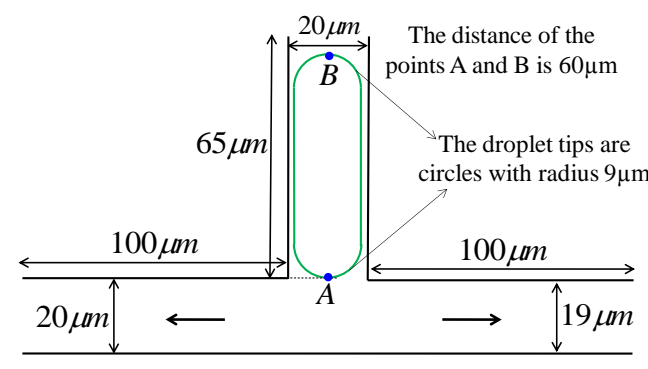

Fig. 1. Geometry of the T-junction with unequal widths.

\section{NUMERICAL SIMULATION}

The flow is incompressible. The continuity and momentum equations are as follows (White 2011):

$$
\frac{\partial u_{i}}{\partial x_{i}}=0 \quad \rho\left(\frac{\partial u_{i}}{\partial t}+u_{j} \frac{\partial u_{i}}{\partial x_{j}}\right)=\rho g_{i}-\frac{\partial p}{\partial x_{i}}+\frac{\partial \tau_{j i}}{\partial x_{j}}
$$

where $u_{i}$ is the vector of velocity and $\tau_{i j}$ is the viscous stress tensor. For power law non-Newtonian fluids, the shear tress tensor can be written as in the following relations:

$$
\tau_{i j}=\eta\left(\frac{\partial u_{i}}{\partial x_{j}}\right) ; \quad \eta=k\left(\frac{\partial u_{i}}{\partial x_{j}}\right)^{n-1}
$$

where $\eta$ is the effective viscosity, $k$ is the consistency index and $n$ is the flow behavior index. In this research, VOF algorithm has been used for simulation of the two-phase flow. $\rho$ and $\mu$ are respectively, the average density and viscosity of the fluid obtained from the following relations:

$$
\begin{aligned}
& \rho=\rho_{c} \phi+\rho_{d}(1-\phi) \\
& \mu=\mu_{c} \phi+\mu_{d}(1-\phi)
\end{aligned}
$$

The subscript $c$ is related to the continuous fluid and the subscript $d$ represents the droplet. $\Phi$ is the volume fraction of the continuous fluid in each computational cell, such that $0 \leq \phi \leq 1$. For the precise location of the boundary, $\phi=0.5$, which is obtained by the Piecewise linear interface reconstruction method. The quantity of $\varphi$ is obtained from the following relation:

$$
\frac{\partial \varphi}{\partial t}+u_{i} \cdot \frac{\partial \varphi}{\partial x_{i}}=0
$$

The discretization of the momentum equations is done by the second order upwind method. The coupling of the pressure and velocity equations is established by the SIMPLEC algorithm. The convergence criterion is such that all residuals should be smaller than 0.001 . The residuals are obtained from the following equation:

$$
R_{X}=\frac{\sum_{P=1}^{N}\left|\sum_{n b} a_{n b} X_{n b}+\Phi-a_{P} X_{P}\right|}{\sum_{P=1}^{N} a_{P} X_{P}}
$$

where $X$ is a general variable in the cell $P$ and $N$ is the total number of cells in domain. $n b$ refers to the neighboring cells of the cell $P$, and $\Phi$ is the constant part of the source term $\left(S=S_{c}+S_{p} X\right)$ and the boundary conditions.

The grid independency is done by solving the 3D breakup problem. Droplet profiles at the moment of deforming in the center of the junction and in different grid sizes were compared. The comparison results are presented in Fig. 2.

As shown in Fig. 2, for the grid with more than 104880 nodes, the results are independent of the grid.

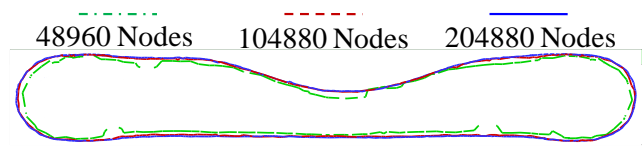

Fig. 2. Grid independency (3D simulation) for the moment at which the droplet is deformed in the center of the junction.

Bretherton (Bretherton 1961) presented an analytical solution for the velocity of a floating droplet in the main fluid moving through a channel. The Bretherton relation is $U=\bar{U}\left(1+1.29\left(3 \mu_{c} U / \sigma\right)^{2 / 3}\right)$ , where $U$ is the velocity of the droplet, and $\sigma, \mu_{c}$ and $\bar{U}$ are the surface tension between the two fluids, the viscosity, and the average velocity of the continuous fluid, respectively. In order to validate the 3D simulation of the present paper, the problem of Bretherton is simulated and its results are compared with the analytical relation of Brwetherton, as can be seen in Fig. 3. This showed a very good agreement (for the Bretherton problem and the grid independency, the fluid viscosity of the 
droplet and continuous fluid is considered 0.001Pa.s and 0.00125Pa.s, respectively).

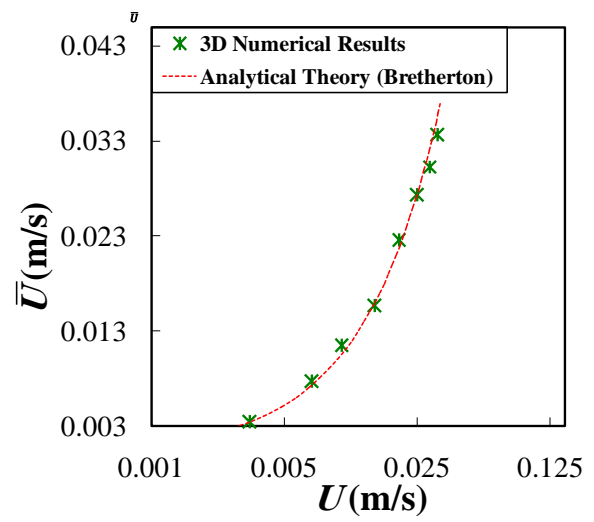

Fig. 3. Comparison of Bretherton's analytical relation (Bretherton 1961) for the velocity of the droplet moving in a channel with the present 3D numerical study.

\section{ANALYTICAL SOLUTiON}

In this research, an analytical solution for a $\mathrm{T}$ junction with unequal-width branches has been done. One of the novelties of this research is presenting an analytical theory for the formulation of some nonNewtonian droplet breakup parameters. Also, a 3D numerical simulation is done to investigate further parameters of the breakup process. It should be noted that there are a few analytical solutions for the breakup process (e.g. Leshansky and Pismen 2009; Bedram et al. 2015b). Each of these analytical solutions presents a few parameters of the breakup process. In other words, an analytical solution cannot formulate all parameters of the breakup process. However, these analytical solutions are very useful and important because they present the exact behavior of the quantities and can be used as the benchmark problems. Therefore, the analytical solution of this paper formulates some breakup process parameters. Further parameters are investigated using a 3D numerical simulation.

The geometry of the problem is a T-junction that has branches with a rectangular cross section (Fig. 4). Figure 1 shows the lengths and widths of the channels. Depth of the geometry (in the direction perpendicular to the paper) is $6 \mu \mathrm{m}$.

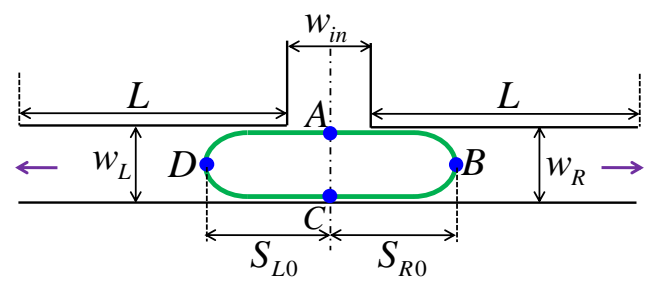

Fig. 4. Geometry of the T-junction with unequal width branches.
For the non-Newtonian fluid that flows within a channel, the Reynolds number is defined as $\operatorname{Re}=\rho U^{2-n} D_{h}^{n} /\left(8^{n-1} k\left(c_{1} / n+c_{2}\right)^{n}\right)$.

Where $\rho$ is the fluid density, $U$ is the average velocity of the fluid in the tube, $k$ is the consistency index, $D_{h}=\frac{4 A}{P}$ the hydraulic diameter, and $n$ is the non-Newtonian flow behavior index. Also, $c_{1}$ and $c_{2}$ are functions based on the shape of the cross-section of the channel. On the other hand, for a non-Newtonian fluid inside the channel, the friction factor will be as $f=64 / R e$ (Muzychka and Edge 2008).

The pressure of the fluid at the system outlet is equal to the ambient pressure. Therefore, the pressure drop in the two sub branches will be the same ( $\Delta P_{L}=\Delta P_{R}$ ) the subscripts $R$ and $L$ represent the right and left branches, respectively. The pressure drop of the fluid inside a channel of the length $L$ will be in the form $\Delta P=\rho f\left(L / D_{h}\right)\left(U^{2} / 2\right)$, such that by substituting $f=64 / R e$ and $D_{h}=\frac{4 A}{P}$ the $\Delta P_{L}=\Delta P_{R}$ have:

$$
\begin{aligned}
& \frac{L_{L} U_{L}^{n} P_{L}^{n+1}\left(c_{1} / n+c_{2}\right)_{L}^{n}}{A_{L}^{n+1}}=\frac{L_{R} U_{R}^{n} P_{R}^{n+1}\left(c_{1} / n+c_{2}\right)_{R}^{n}}{A_{R}^{n+1}} \\
& \rightarrow \frac{U_{R}}{U_{L}}=\left(\frac{L_{L}}{L_{R}}\right)^{\frac{1}{n}}\left(\frac{A_{R}}{A_{L}} \frac{P_{L}}{P_{R}}\right)^{\left(1+\frac{1}{n}\right)} \frac{\left(c_{1} / n+c_{2}\right)_{L}}{\left(c_{1} / n+c_{2}\right)_{R}}
\end{aligned}
$$

Where $U$ is the continuous fluid velocity in the channel, $L$ is the channel length, $A$ and $P$ are the area and perimeter of the branches cross sections, respectively, and the subscripts $R$ and $L$ represent the right and left branches, respectively.

The geometry of the problem is such that after the breakup of the initial droplet, the smaller droplet enters the right branch and the larger droplet enters the left one. Therefore, the "volume ratio" quantity is defined as $\forall_{R} / \forall_{L}$, where $\forall_{R}$ and $\forall_{L}$ are the volume of the droplet entering into the right and left branches, respectively. In the case of droplet breakup in the T-junction, it can be assumed that the ratio of the volume of the two generated droplets is equal to that of the volumetric flow rate of the two junction branches (Link et al. 2004). So, we have $\forall_{R} / \forall_{L}=Q_{R} / Q_{L}$, where $Q_{R}$ and $Q_{L}$ are the volumetric flow rate in the right and left branches, respectively. Therefore, the ratio of the volume of the two droplets entering the branches (which is equal to the ratio of the two branches flow rate) will be as follows:

$$
\begin{aligned}
& \frac{\forall_{R}}{\forall_{L}}=\frac{Q_{R}}{Q_{L}}=\frac{A_{R} U_{R}}{A_{L} U_{L}} \\
& \rightarrow \frac{Q_{R}}{Q_{L}}=\left(\frac{L_{L}}{L_{R}}\right)^{\frac{1}{n}}\left(\frac{A_{R}}{A_{L}}\right)^{\left(2+\frac{1}{n}\right)}\left(\frac{P_{L}}{P_{R}}\right)^{\left(2+\frac{1}{n}\right)} \frac{\left(c_{1} / n+c_{2}\right)_{L}}{\left(c_{1} / n+c_{2}\right)_{R}}
\end{aligned}
$$


Inlet flow rate $\left(Q_{i n}\right)$ equal to the sum of the branches flow rate $\left(Q_{\mathrm{in}}=Q_{\mathrm{R}}+Q_{\mathrm{L}}\right)$. Therefore, we have:

$$
\frac{Q_{L}}{Q_{i n}}=\frac{1}{1+\left(\frac{Q_{R}}{Q_{L}}\right)} \quad \frac{Q_{R}}{Q_{i n}}=\frac{1}{1+\left(\frac{Q_{R}}{Q_{L}}\right)^{-1}}
$$

We can calculate the ratio of the right branch velocity to the inlet fluid velocity by using the relation $Q=U A$, as follows:

$$
\frac{U_{R}}{U_{\text {in }}}=\frac{Q_{R}}{Q_{\text {in }}} \frac{A_{\text {in }}}{A_{R}} \quad \frac{U_{L}}{U_{\text {in }}}=\frac{Q_{L}}{Q_{\text {in }}} \frac{A_{\text {in }}}{A_{L}}
$$

For channels with a rectangular cross section, the aspect ratio, indicated with $\varepsilon$, is defined as the smaller side to the larger side of cross section and the $c_{1}$ and $c_{2}$ values are obtained based on the aspect ratio. According to the geometric sizes (Fig. 1), we have $\varepsilon_{R}=0.3158, \quad \varepsilon_{L}=0.3, \quad P_{R}=5 \times 10^{-5} \mathrm{~m}$, $P_{L}=5.2 \times 10^{-5} \mathrm{~m}, A_{R}=1.14 \times 10^{-10} \mathrm{~m}^{2}, A_{L}=1.2 \times 10^{-10} \mathrm{~m}^{2}$, $A_{\text {in }}=1.2 \times 10^{-10} \mathrm{~m}^{2}$, and $L_{R}=L_{L}=100 \times 10^{-6} \mathrm{~m}$ that $P$ and $A$ are the perimeter and area of the cross section, respectively. $L$ is the branch length. With the aspect ratio $(\varepsilon)$ for each branch, the values $c_{1}$ and $c_{2}$ become as $\left(c_{1}\right)_{R}=0.29389, \quad\left(c_{1}\right)_{L}=0.29914$, $\left(c_{2}\right)_{R}=0.78932$ and $\left(c_{2}\right)_{L}=0.79536$ (Muzychka and Edge 2008). In this research $n=1.1$. Therefore, by substituting the geometric parameters in Eqs. (7) and (8), we have $U_{R} / U_{L}=0.9872$ and $Q_{R} / Q_{L}=0.93784$. Also, by substituting these values in Eqs. (9) and (10), we will obtain $Q_{R} / Q_{i n}=0.484$, $Q_{L} / Q_{i n}=0.516, U_{R} / U_{i n}=0.5095$ and $U_{L} / U_{i n}=0.516$.

Figure 5 presents the analytical and numerical results of the ratio of the right branch flow rate to the left branch flow rate $\left(Q_{R} / Q_{L}\right)$ the right branch flow rate to the inlet flow rate $\left(Q_{R} / Q_{i n}\right)$ and the left branch flow rate to the inlet flow rate $\left(Q_{L} / Q_{i n}\right)$ during the droplet breakup process. As can be seen, the ratio of the branch flow rate is constant in time. This is useful for industrial applications of the breakup of droplets since, in the pharmaceutical, chemical and petrochemical industries, the generation of a large number of equal sized droplets is important. On the other hand, it has been previously stated that the ratio of the volume of the generated droplets in the geometry of this paper is equal to that of the flow rate of the right branch to the left one. Now, if the ratio of the flow rate of branches remains constant over time, the ratio of the volume of generated droplets will not change with time, which is a desirable phenomenon. In addition, according to the figure, the flow rate of the right branch is about six percent lower than that of the left one. This is because the right branch, due to its lower width, has higher hydraulic resistance; so, a smaller percent of the flow rate of the inlet channel enters the right branch and the greater percent enters the left one. According to the figure, the analytical and numerical results of the flow rate ratio of the branches have a very good agreement.

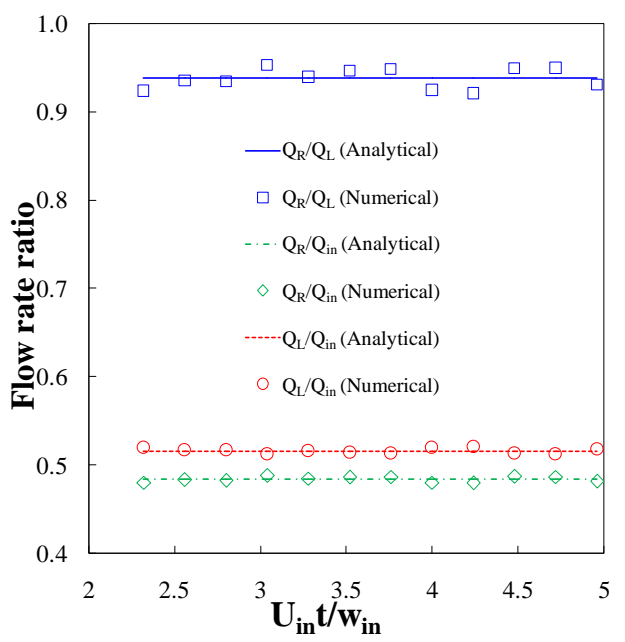

Fig. 5. Analytical and numerical results of the flow rate ratio of the branches during the droplet breakup process.

Figure 6 represents the analytical and numerical results of the right branch velocity to the left branch velocity $\left(U_{R} / U_{L}\right)$ and right branch velocity to the inlet channel velocity $\left(U_{R} / U_{i n}\right)$ and the left branch velocity to the inlet channel velocity $\left(U_{L} / U_{i n}\right)$ during the droplet breakup process. As can be seen, the fluid velocity ratio in branches does not change over time. As can be seen, fluid velocity in the right branch is about one percent lower than that the left one. The difference between the velocity of the right and left branches (1\% according to Fig. 6) is less than that between the flow rate of the right and left branches (6\% according to Fig. 5). It is since the flow of the right branch is less than that of the left one. So, according to Eq. (10), the percent of difference in the right and left branches velocity will be less than that between their flow rates. It can also be observed that the analytical and numerical results of the ratio of the branches velocities have a very good agreement.

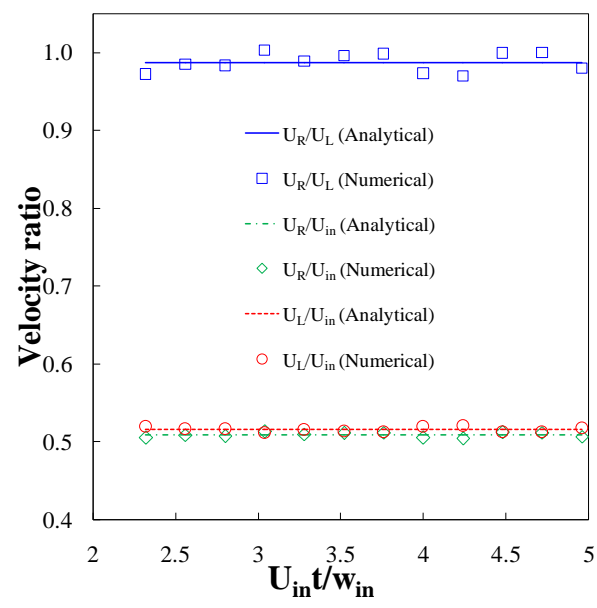

Fig. 6. Analytical and numerical results of the velocity ratio of the branches during the droplet breakup process. 
As the droplet deforming occurs at the junction center, at a specific moment, the droplet becomes as shown in Fig. 4. This moment is considered as the initial time of the breakup process $(t=0)$. As can be seen in Fig. 4, the volume of the droplet of the right branch $\left(\forall_{R}\right)$ is equal to that of the ABCA part. The volume of droplets in the left branch $\left(\forall_{L}\right)$ is equal to that of the ADCA part. As already stated, for the droplet breakup in a T-junction, we can assume that the ratio of the volume of the two generated droplets in the branches is equal to the flow rate ratio in the two branches (Link et al. 2004). So, as can be seen in Fig. 4, we have $\forall_{R} / \forall_{L}=Q_{R} / Q_{L}$. The value $Q_{R} / Q_{L}$ was obtained as shown in Eq. (8). We know that the sum of $\forall_{L}$ and $\forall_{R}$ is equal to the total volume of the droplet $\left(\forall_{i n}\right)$. Therefore, we have $\forall_{L} / \forall_{i n}=Q_{L} / Q_{\text {in }}$ and $\forall_{R} / \forall_{i n}=Q_{R} / Q_{i n}$, which can be obtained from Eq. (9).

Our previous paper (Bedram et al. 2015a) indicated that in the droplet breakup problems, 2D and 3D results are very close. So, to shorten the analytical computations, we used the $2 \mathrm{D}$ geometry for the rest of the analytical solution.

Dimensions of the 2D geometry are exactly as shown in Fig. 1. Several studies have already shown that the end of the droplet (i.e., the location of the points B and D in Fig. 4) is in the form of semicircles whose diameter is approximately equal to the channel width (Leshansky and Pismen 2009). So, by applying Eq. (9) and considering Fig. 4, we have:

$$
\begin{aligned}
& \frac{\forall_{R}}{\forall_{\text {in }}}=\frac{Q_{R}}{Q_{i n}} \\
& \rightarrow \frac{\left(S_{R 0}-\frac{w_{R}}{2}\right) w_{R}+\frac{\pi w_{R}^{2}}{8}}{\left(S_{i n}-w_{i n}\right) w_{i n}+\frac{\pi w_{i n}^{2}}{4}}=\frac{1}{1+\left(\frac{Q_{R}}{Q_{L}}\right)^{-1}} \\
& \rightarrow \frac{S_{R 0}}{w_{R}}=\frac{\left(\frac{S_{i n}}{w_{i n}}-1\right)+\frac{\pi}{4}}{\left[1+\left(\frac{Q_{R}}{Q_{L}}\right)^{-1}\right]\left(\frac{w_{R}}{w_{i n}}\right)^{2}}-\frac{\pi}{8}+\frac{1}{2}
\end{aligned}
$$

where $S_{\text {in }}$ is the initial length of the droplet before reaching the junction center. Similarly, for the left branch, we have:

$$
\begin{aligned}
& \frac{\forall_{L}}{\forall_{i n}}=\frac{Q_{L}}{Q_{i n}} \\
& \rightarrow \frac{\left(S_{L 0}-\frac{w_{L}}{2}\right) w_{L}+\frac{\pi w_{L}^{2}}{8}}{\left(S_{i n}-w_{i n}\right) w_{i n}+\frac{\pi w_{i n}^{2}}{4}}=\frac{1}{1+\left(\frac{Q_{R}}{Q_{L}}\right)} \\
& \rightarrow \frac{S_{L 0}}{w_{L}}=\frac{\left(\frac{S_{i n}}{w_{i n}}-1\right)+\frac{\pi}{4}}{\left[1+\left(\frac{Q_{R}}{Q_{L}}\right)\right]\left(\frac{w_{L}}{w_{i n}}\right)^{2}}-\frac{\pi}{8}+\frac{1}{2}
\end{aligned}
$$

A few moments after the start of the breakup process, the droplet shape changes to that represented in Fig. 7. When the droplet enters branches, it fills the branch space. The velocity of the point B (Fig. 7) is equal to that of the continuous fluid in the right branch.

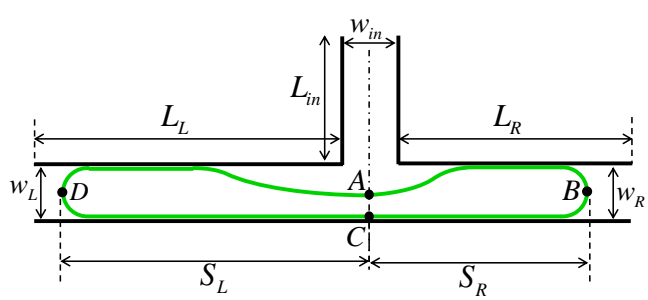

Fig. 7. Geometry of the T-junction with unequal widths. The deformed droplet is shown at the center of the junction.

So, we can calculate the length of the droplet in the right $\left(S_{R}\right)$ and left $\left(S_{L}\right)$ branches, as follows:

$$
\begin{aligned}
& S_{R}=S_{R 0}+U_{R} \times t \rightarrow \frac{S_{R}}{w_{R}}=\frac{S_{R 0}}{w_{R}}+\frac{U_{R}}{U_{i n}} \frac{U_{i n} t}{w_{R}} \\
& S_{L}=S_{L 0}+U_{L} \times t \rightarrow \frac{S_{L}}{w_{L}}=\frac{S_{L 0}}{w_{L}}+\frac{U_{L}}{U_{\text {in }}} \frac{U_{i n} t}{w_{L}}
\end{aligned}
$$

$U_{R} / U_{\text {in }}$ and $U_{L} / U_{\text {in }}$ can be calculated from Eq. (10). Also, $S_{R 0} / w_{R}$ and $S_{L 0} / w_{L}$ can be calculated from Eqs. (11) and (12), respectively.

In the 2D geometry, the depth of the system (in a direction perpendicular to the paper) is infinite; so, we have $\varepsilon_{R}=\varepsilon_{L}=0, \quad P_{R}=P_{L}=2 Z, \quad A_{R}=19 \times 10^{-6} Z$, $A_{L}=A_{\text {in }}=20 \times 10^{-6} Z$ and $L_{R}=L_{L}=100 \times 10^{-6} \mathrm{~m}$, where $Z$ is the depth of geometry in the direction perpendicular to the paper. $P$ and $A$ are also the perimeter and area of the cross section, respectively. With the aspect ratio $(\varepsilon)$ for each branch, the values $c_{l}$ and $c_{2}$ become as $\left(c_{1}\right)_{R}=\left(c_{1}\right)_{L}=0.4938$ and $\left(c_{2}\right)_{R}=\left(c_{2}\right)_{L}=0.99275$ (Muzychka and Edge 2008). Therefore, by substituting the geometric parameters in Eqs. (7) and (8), we have $U_{R} / U_{L}=0.90672$ and $Q_{R} / Q_{L}=0.86138$. Also, by substituting these values in Eqs. (9) and (10), we will obtain $Q_{R} / Q_{i n}=0.46276$, $Q_{L} / Q_{\text {in }}=0.53724, \quad U_{R} / U_{\text {in }}=0.48712 \quad$ and $U_{L} / U_{i n}=0.53724$. In this paper, $S_{\text {in }}=3.333 \times w_{\text {in }}=66.66 \times 10^{-6} \mathrm{~m}$. Therefore, by substituting these values in Eqs. (11) and (12), we will obtain $S_{R 0} / w_{R}=1.70627, \quad S_{L O} / w_{L}=1.78263$. Finally, by substituting these values in Eq. (13), we have $\quad S_{R} / w_{R}=1.70627+5127.6 \times t \quad$ and $S_{L} / w_{L}=1.78263+5372.4 \times t$.

Figure 8 presents the analytical and numerical results of the droplet length in the right branch $\left(S_{R} / w_{R}\right)$ the length of the droplet in the left branch $\left(S_{L} / w_{L}\right)$ and the whole length of the droplet $\left(S_{R} / w_{R}+S_{L} / w_{L}\right)$ during the breakup process. As can be seen, the length of the droplet in each of the branches and the total length of the droplet increase linearly with time. The reason for this, as shown in 
Fig. 7, is that the length of the droplet is equal to the distance between the point $\mathrm{B}$ and the center of the junction. On the other hand, the velocity of the point $\mathrm{B}$ is equal to that of the continuous fluid in the right branch. Therefore, the point B moves with a constant velocity, so its distance from the junction center (i.e. the length of the droplet) will increase linearly with time.

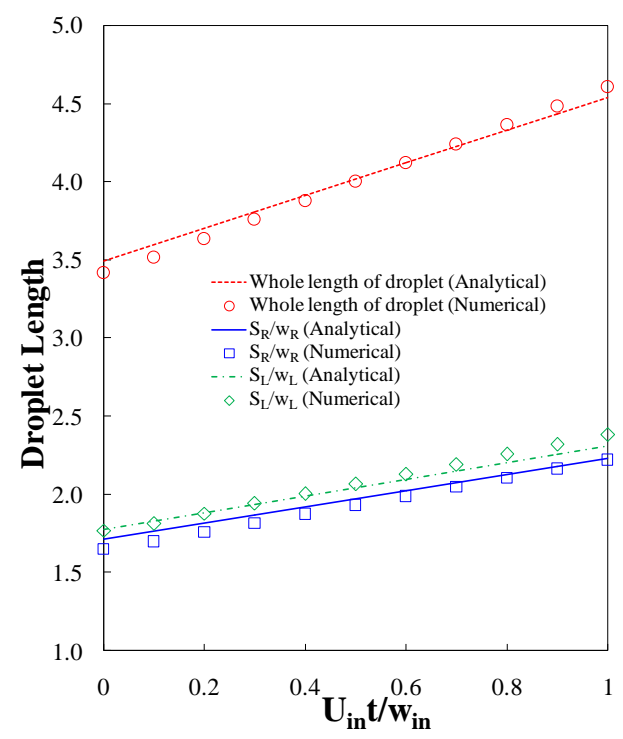

Fig. 8. Analytical and numerical results of the droplet length in the right branch $\left(S_{R} / w_{R}\right)$, the droplet length in the left branch $\left(S_{L} / w_{L}\right)$, and the whole length of the droplet $\left(S_{R} / w_{R}+S_{L} / w_{L}\right)$ during the breakup process.

It is also seen that the length of the droplet in the right branch is slightly less than that of the droplet in the left branch, because the right branch, due to its less width, has a higher hydraulic resistance; therefore, a less volume of droplets enters this branch. According to the figure, our analytical and numerical results have a very good agreement.

Figure 9 shows the breakup process of the droplet in the T-junction with unequal width branches. The tip of the droplet is circular (we used this assumption in our analytical theory). The upper surface of droplet is an arc.

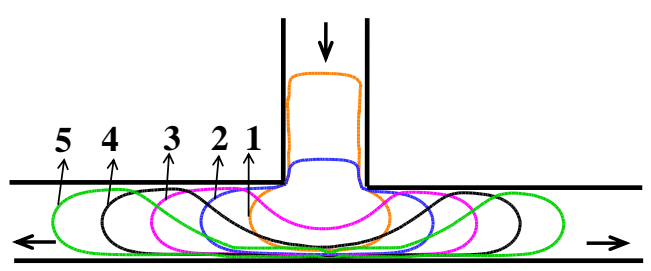

Fig. 9. Breakup process of the droplet in the T-junction with unequal width branches.

\section{RESULTS AND DISCUSSION}

In this section, 3D numerical simulation results are reported in four different droplet modes. These include:

1- Before reaching the droplet to the center of the junction

2- in the early moments of droplet deformation

3- at the last moments of droplet deformation

4- $\quad$ after the droplet breakup

One of the applications of droplet breakup is mixing different materials in a droplet and then breaking it to smaller parts. Therefore, the motion of droplet in a straight channel should be investigated. So, in this paper, the numerical results are reported for the state that the droplet is in the inlet channel.

We define dimensionless quantities for the horizontal and vertical axes based on these relations:

$$
\Phi=x / w_{\text {in }} \quad \Gamma=y / w_{\text {in }}
$$

Here, $w_{\text {in }}$ is the inlet channel width. The droplet breakup process is shown in Fig. 10.

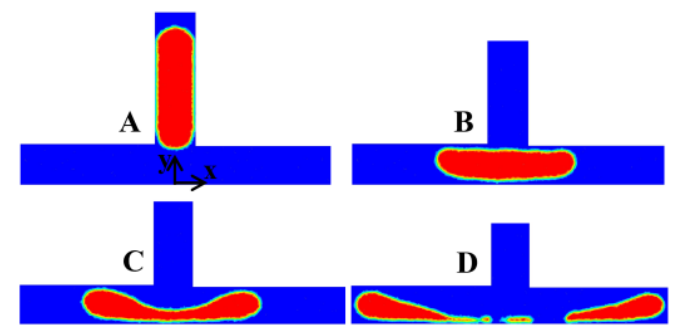

Fig. 10. Droplet breakup process.

During the breakup process, the fluid pressure decreases in some locations of the system. This causes the fluid boiling temperature to decrease. Therefore, in the biological or chemical applications of droplet breakup process, some materials inside droplet may evaporate. Therefore, investigation of the pressure distribution during the droplet breakup process is important.

Figure 11 represents the distribution of fluid pressure in five different sections of the droplet before the droplet reaches the center of the junction. The droplet location is shown in this Figure. As can be seen, the pressure of the fluid is constant along the width of the input channel, because there is no flow along the width of the channel; so, according to the NaiverStokes equation, there is no pressure gradient. For each curve of Fig. 11, it can be seen that the pressure of the fluid near the wall is slightly less than that at the midpoints of the channel. This is also indicated by the dotted circles in Fig. 11. The reason is that the points of the beginning and end of each curve are in the continuous fluid and the other points are inside the droplet. On the other hand, the pressure of the fluid inside the droplet (which is due to the effects of surface tension) is more than that outside it 
$(\Delta P=\sigma / r)$. Figure 11 also shows that the fluid pressure decreases along the inlet channel length because the flow exists in the pipe and the pressure is reduced along the pipe due to the effects of viscous flow.

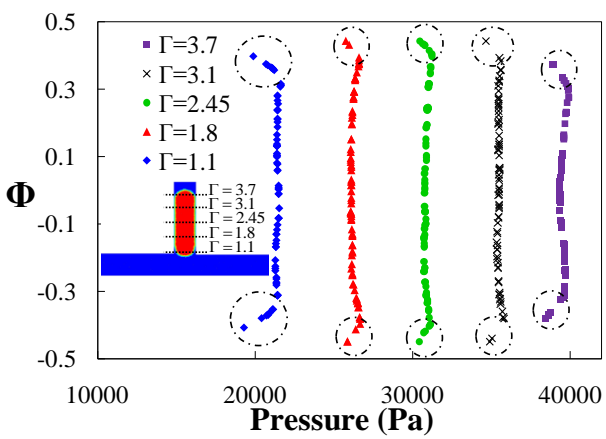

Fig. 11. Fluid pressure in five different sections of the droplet, before reaching the center of the junction.

For mixing some materials, one can inject them to a droplet. The locations inside the droplet that have more velocity gradients have better mixing performances. Therefore, the materials should be injected in these locations. So, investigation of fluid velocity distribution during the breakup process is needed.

Figure 12 shows the velocity of the fluid in five different sections of the droplet. The droplet location is shown in the Figure. As can be seen, the fluid velocity distribution is parabolic. The reason is that the flow inside the inlet channel is laminar (Reynolds number is less than 10)

In the section $\Gamma=1.1$, the tip of the droplet is approaching the junction center; so, the fluid velocity in these regions is different from other regions of the droplet. This issue is also illustrated in Fig. 12.

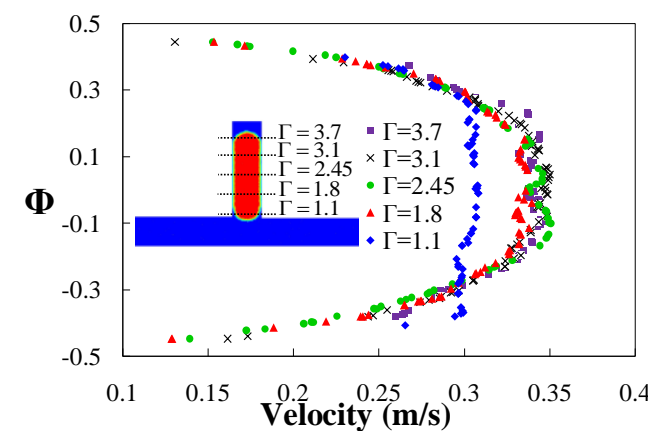

Fig. 12. Fluid velocity in five different sections along the droplet.

The amount of vorticity in five different sections of the droplet is shown in Fig. 13. The droplet location is displayed in this Figure. As the amount of vorticity is increased, the velocity gradient is increased within the droplet too. So, mixing inside the droplet is increased, which is desirable in industrial applications such as pharmaceutical and chemical industries. In regions near the wall, the vorticity increases because the velocity gradient is high. Therefore, the mixing of the material inside the droplet is increased near the wall.

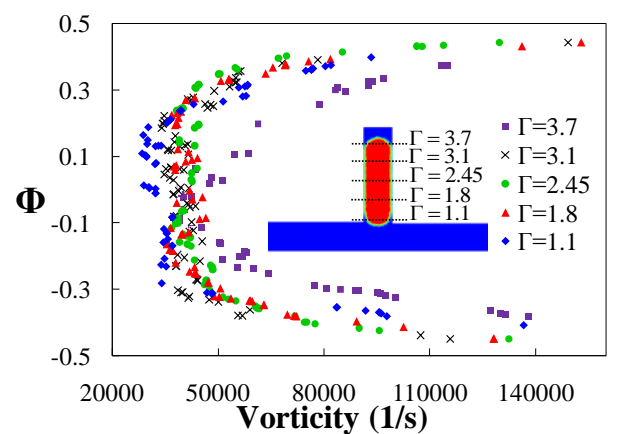

Fig. 13. Vorticity in five different sections along the droplet.

Figure 14 depicts the fluid pressure in the five different sections of the droplet in the initial moments of the droplet deformation in the center of the junction. As can be seen, the pressure of the fluid in the regions near the center of the junction $(\Phi=0)$ has the highest value; by moving away from the center of the junction, the fluid pressure is decreased. It is because the flow from the center of the junction to the branches is established, and due to the presence of surface roughness and according to the momentum equation, the pressure will decrease in this direction. Also, due to the negligible fluid velocity component along the width of the channel, the pressure gradient is negligible in this direction. Therefore, the pressure of the fluid in width of the channel is constant; however, in the curve $\Phi=0$, the pressure of the fluid is not constant, which is because this section is closer to the input channel. Due to surface tension, the pressure of the fluid inside the droplet is slightly higher than that outside it. For this reason, as can be seen in Fig. 14, the beginning and end of each curve (indicated by the dotted ellipse) have lower pressure.

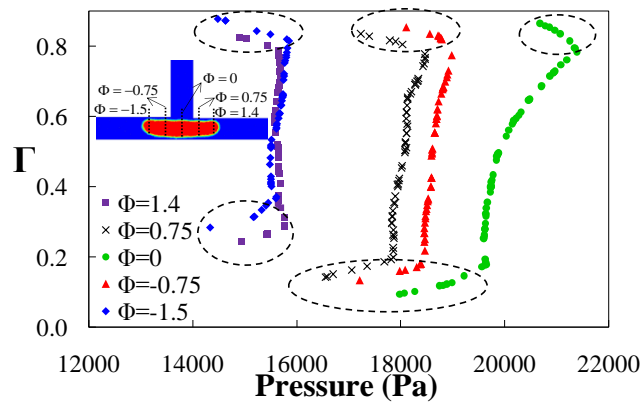

Fig. 14. Fluid pressure in five different sections of the droplet, at the initial moment of droplet deformation in the center of the junction. 
Figure 15 shows the velocity of the fluid in five different sections of the droplet at the initial moments of the droplet deformation in the center of the junction. The regions near the point $\mathrm{C}$ in Fig. 4 act like a stagnation point, because in the center of the junction, the fluid passes from the two sides to the two branches. Therefore, in regions near $\mathrm{C}$, fluid velocity will be low. For this reason, it is seen in Fig. 15 that the curve of $\Phi=0$ has a velocity near zero in the regions near the wall.

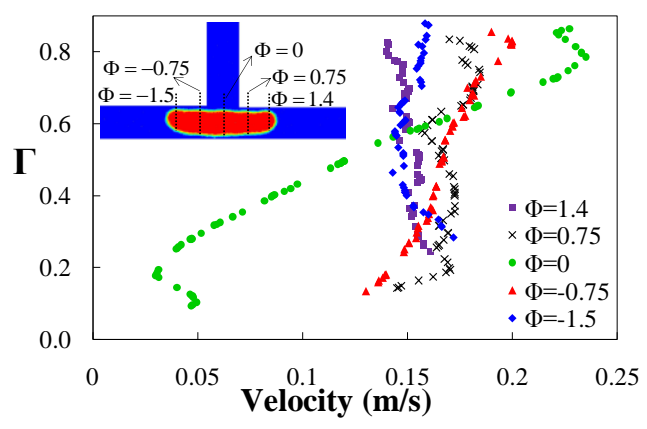

Fig. 15. Fluid velocity in five different selected sections of the droplet, at the initial moment of droplet deformation in the center of the junction.

Figure 16 shows vorticity magnitude in five different droplet sections at the initial moments of droplet deformation in the center of the junction. In all droplet sections, regions near the droplet surface have higher vorticity; so, mixing the materials of the droplet in these regions is increased. By comparing Figs. 13 and 16, we could find that the vorticity values at the initial moments of the droplet deformation are less than the moment at which the droplet has not yet reached the center of the junction. According to these two graphs, the vorticity values before the droplet reaches the center of junction are between $30,000 \mathrm{~s}^{-1}$ and $150,000 \mathrm{~s}^{-1}$. Also, the vorticity values at the initial moments of the droplet deformation are between $10,000 \mathrm{~s}^{-1}$ and $11,000 \mathrm{~s}^{-1}$.

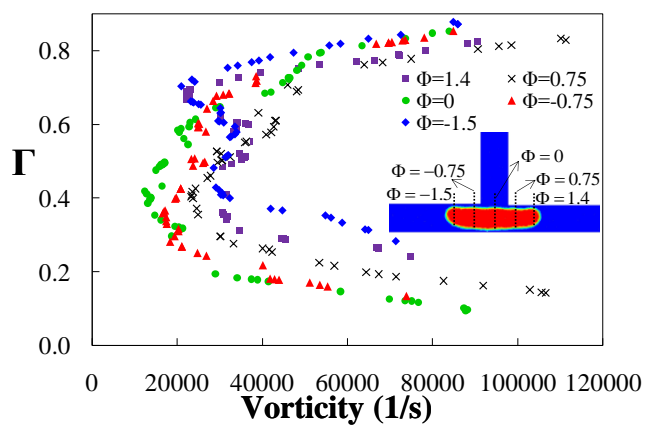

Fig. 16. Vorticity in five different sections along the droplet at the initial moment of droplet deforming in the center of the junction.

Figure 17 shows the fluid pressure in five different sections of the droplet at the final moment of droplet deformation in the center of the junction. As can be seen, in each section, the fluid pressure is almost uniform, which is due to the absence of the component of the fluid velocity along the channel width, leading to the absence of the pressure gradient in this direction. As can be seen in Fig. 17, the sections closer to the output of each branch have lower pressure, because fluid pressure decreases along the branches (due to the effects of viscosity and surface roughness). Also, due to surface tension, the pressure of the fluid inside the droplet is slightly higher than that outside it. For this reason, it can be seen that the beginning and end of each curve (as indicated by the dotted ellipse) have lower pressure.

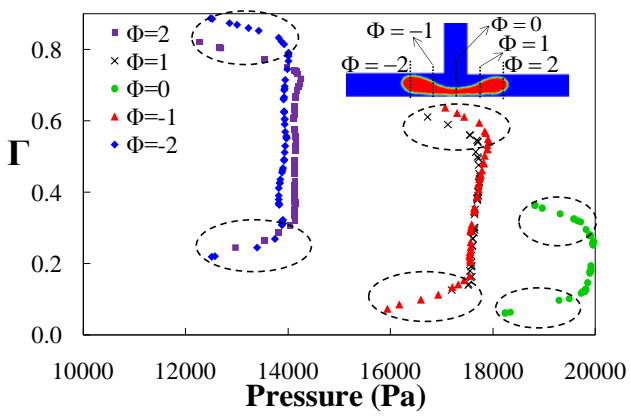

Fig. 17. Fluid pressure in five different sections, at the final moments of the droplet deformation.

Furthermore, by comparing Figs. 14 and 18, we can find that the pressure of the fluid in the center of the junction at the initial moments of droplet deformation (Fig. 14) is an average of $20000 \mathrm{~Pa}$; meanwhile, the pressure of the fluid in the center of the junction at the final moments of the deformation (Fig. 17) is an average of $20000 \mathrm{~Pa}$ (in this analysis, the regions shown in Figs. 14 and 17 with dotted ellipses should not be considered, because these regions of the fluid are close to the droplet surface). Therefore, the fluid pressure at a specific point in the system does not change over time.

Figure 18 shows the velocity of the fluid in five different sections of the droplet at the final moments of the droplet deformation in the center of the junction. As can be seen, the $\Phi=0$ curve showed very low velocity at places near zero. This is because in the center of the junction, the points near the bottom wall (regions near the point $\mathrm{C}$ in Fig. 4) must have a negligible velocity, because the flow in this region is divided into two parts and in two opposite directions.

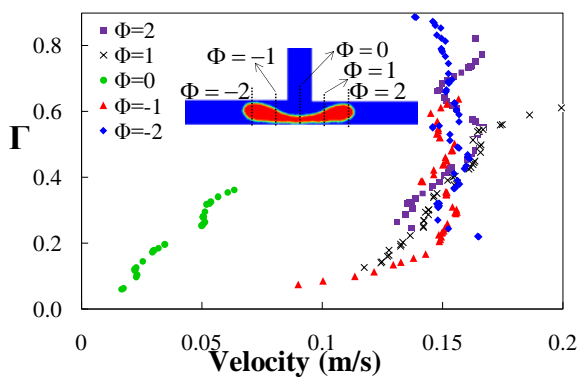

Fig. 18. Fluid velocity in five different sections along the droplet length at the final moments of droplet deformation in the center of the junction. 
Figure 19 shows the vorticity magnitude in five different droplet sections at the final moment of the droplet deformation in the center of the junction. As can be seen, the regions near the droplet surface have higher vorticity; so, the vorticity values near the droplet surface are 3 to 5 times more than those in the middle of the channel. Therefore, the mixing of the material of the droplet in these regions is increased. As can be seen, the vorticity distribution in all sections is almost parabolic.

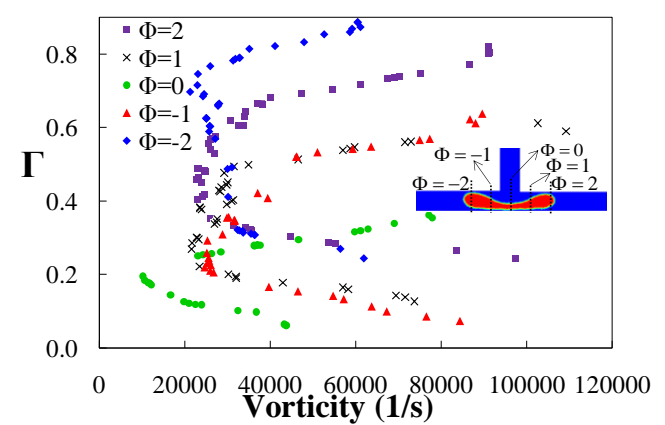

Fig. 19. Vorticity in five different sections along the droplet at the final moment of droplet deformation in the center of the junction.

Figure 20 shows the fluid pressure in six different sections after the droplet breakup. According to this figure, the sections near the center of the junction have higher pressure (due to the flow from the junction center to the branches output). Also, the fluid pressure at each section is uniform (due to the absence of the flow along the channel width). Some industrials that employ microfluidic processes use fluids with a low boiling point. In these cases, the pressure of the fluid must not be reduced because it may then reach the vapor pressure; so, part of the fluid is evaporated and the process is disrupted. In this study, the minimum pressure can be seen in Fig. 20 , in the curves $\Phi=3.65$ and $\Phi=-3.6$ at the points near the droplet surface. Therefore, the choice of fluid type for microfluidic systems should be carefully considered.

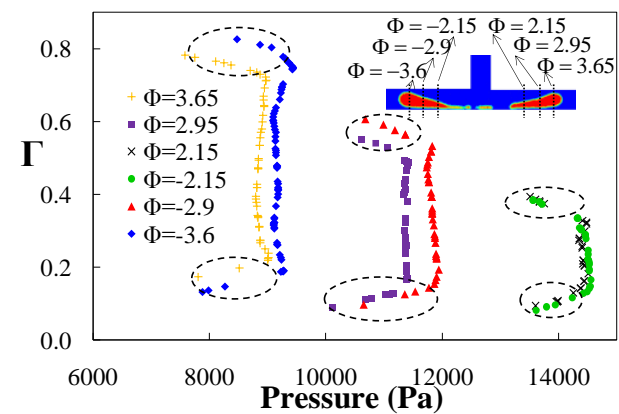

Fig. 20. Fluid pressure in six different sections of the droplet, after the droplet breakup.

Figure 21 shows the velocity of the fluid in six different sections after the droplet breakup. As can be seen in Fig. 21 at dimensionless vertical place
0.37 , the curves $\Phi=-2.15$ and $\Phi=2.15$ have the highest velocity. The reason for this is that in $\Phi=-2.15$ and $\Phi=2.15$ sections, the dimensionless vertical point 0.37 corresponds to the upper droplet surface. On the other hand, at the moment when the droplet is in the state of Fig. 21, the shape of each droplet in each branch is similar to the airfoil. Therefore, the droplet tends to circle itself due to the effects of surface tension. So, the upper surface of the droplet takes a higher velocity to bring it to the upper wall, forming a circular droplet.

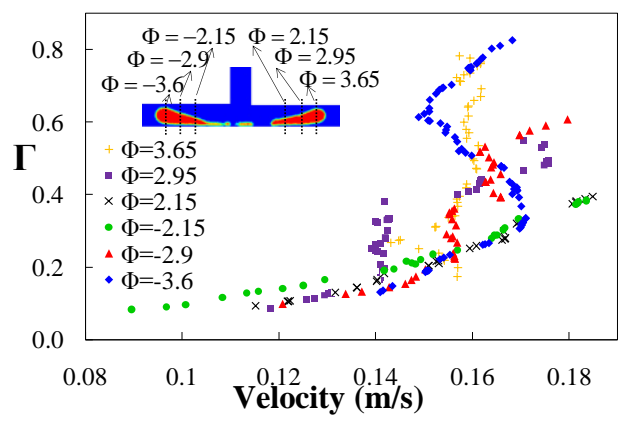

Fig. 21. Fluid velocity in six different sections from the beginning to the end of droplet after the droplet breakup.

Figure 22 shows vorticity magnitude in six different sections of the droplet after the droplet breakup. By comparing Figs. 16, 19, and 22, we can find that the greatest vorticity occurs after the droplet breakup. In other words, at the first moments of droplet deformation (Fig. 16), the vorticity in different sections of the droplet is between $10000 \mathrm{~s}^{-1}$ and $110000 \mathrm{~s}^{-1}$; at the final moments (Fig. 19), the vorticity in different sections of the droplet is between $10000 \mathrm{~s}^{-1}$ and $110000 \mathrm{~s}^{-1}$. After the droplet breakup (Fig. 22), the vorticity in different sections of the droplet is between $20000 \mathrm{~s}^{-1}$ and $136000 \mathrm{~s}^{-1}$. Therefore, the vorticity increases after breakup. The reason is that after the breakup, the initial shape of the generated droplets is stretched; in a short time, the droplet is deformed, taking a circular shape (similar to Fig. 10-A). Therefore, this rapid deformation of droplet increases the flows of droplet inside, causing the increase of the velocity gradient and vorticity. In addition, according to Fig. 22, the vorticity of the regions near the droplet surface is approximately 3-7 times higher than that the droplet.

Figure 23 shows the effect of non-Newtonian powerlaw index $(n)$ on the fluid pressure. The location of the droplet is shown in Fig. 10-A. The fluid pressure is reported in section $y=49 \mu \mathrm{m}$. As can be seen, the fluid pressure is constant along the channel width due to the absence of the flow along the channel width. Also, the effect of the T-junction with the unequal length is investigated and compared with the T-junction with unequal width branches. The geometry of the T-junction with unequal length branches is as shown in Fig. 1, but the widths of the two branches are equal and the length of the right and left branches are $100 \mu \mathrm{m}$ and $80 \mu \mathrm{m}$, respectively. Figure 23 compares the fluid pressure in the $\mathrm{T}$ junction with unequal width branches and $\mathrm{T}$-junction 


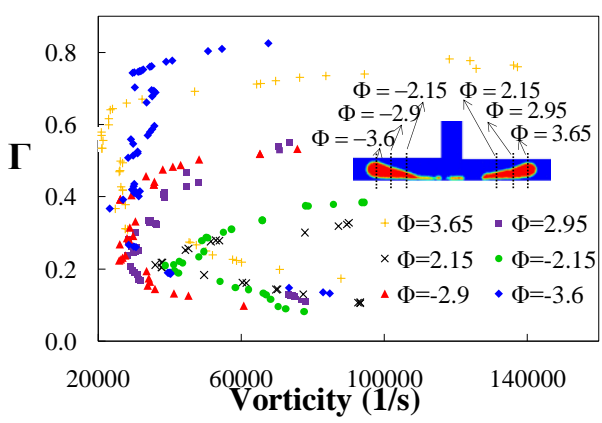

Fig. 22. Vorticity in six different sections from the beginning to the end of the droplet after the breakup.

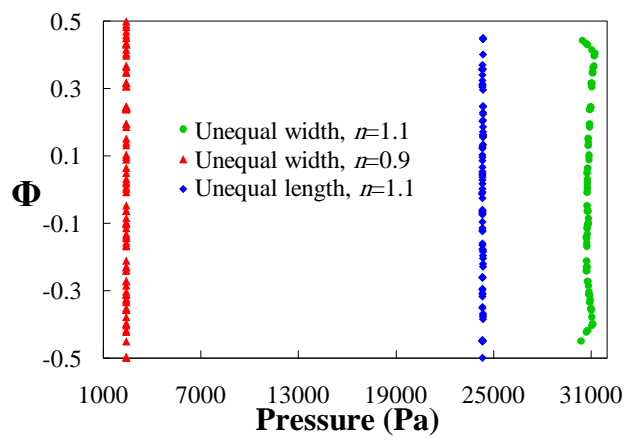

Fig. 23. Effect of the behavior index of non-Newtonian power-law fluid $(n)$ and unequal length of $\mathrm{T}$-junction branches on fluid pressure.

with unequal length branches. As can be seen, the fluid pressure in the T-junction with unequal length branches is less, which is because the left branch of the unequal length T-junction is smaller than that of the unequal width one. Therefore, the hydrodynamic resistance and pressure drop of the unequal length $T$ junction are less than those of the unequal width one. So, the fluid pressure in a specific section of the unequal length T-junction is less than that of the unequal width one.

Figure 24 illustrates the effect of non-Newtonian power-law index $(n)$ and different geometries (unequal length $\mathrm{T}$-junction and unequal width $\mathrm{T}$ junction) on vorticity. The location of the droplet is shown in Fig. 10-A and vorticity is reported in section $\mathrm{y}=49 \mu \mathrm{m}$. As can be seen, the vorticity of $n=1.1$ is more than that of $n=0.9$. Therefore, vorticity increases with $n$. So, to increase mixing inside the droplet, the non-Newtonian power-law index should be increased. Also, this figure illustrates that vorticity is minimum in the center of the channel in both unequal length and unequal width T-junctions. It is since the velocity gradient near the wall is high; so, the vorticity magnitude in these regions has its maximum value.

\section{Conclusion}

In this paper, an analytical investigation and 3D numerical simulation were presented for the breakup

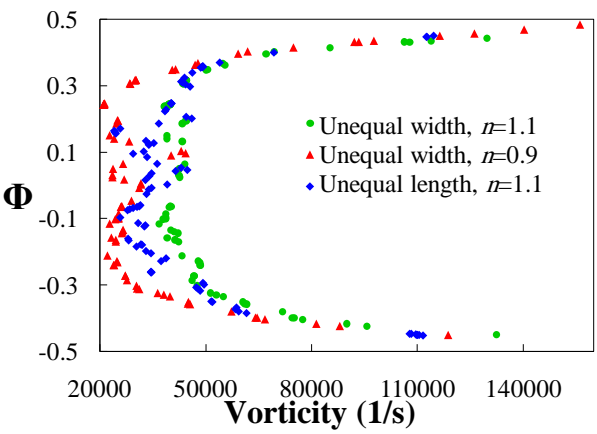

Fig. 24. Effect of the behavior index of non-Newtonian power-law fluid $(n)$ and unequal length of $T$-junction branches on vorticity.

of floating non-Newtonian droplets in a nonNewtonian fluid. The considered geometry was a Tjunction with unequal-width branches that could generate droplets with an unequal size. In the previous researches, neither analytical nor numerical solutions had been considered for the asymmetric breakup of non-Newtonian droplets; so, for the first time, these two cases were performed in this paper. As the geometric advantages of this study, after the generation of new droplets, small and large generated droplets were not mixed; so, there was no need for another process to separate droplets. Grid independency was performed for the 3D numerical simulation. The analytical and 3D numerical simulation results of this research had a very good agreement. In addition, numerical simulation results were compared with the analytical solution of a benchmark problem, observing a good agreement. Numerous quantities such as branch flow rate ratio, branch velocity ratio, the droplet length in each branch, whole length of the droplet, vorticity and pressure during breakup process were investigated. The analytical solution of this research resulted in the values of branch flow rate ratio, branch velocity ratio, droplet length in each branch and whole length of the droplet during the breakup process. The results also showed that the branches flow rate ratio and the fluid velocity ratio of the branches did not change during the breakup process. In addition, it was observed that the length of the droplet in each of the branches and the whole length of the droplet increased linearly during the breakup process. The results of 3D numerical simulation also showed that during the breakup process, the fluid pressure was constant along the channel width. It was also observed that the pressure of the fluid near the droplet surface was lower than that in the middle regions of the droplet. The results also indicated that the velocity distribution of the fluid was parabolic before the droplet reached the junction center. It was also revealed that vorticity values in the regions near the droplet surface were 3 to 7 times higher than those in the middle regions of the droplet, which increased the mixing of the material inside the droplet. Additionally, the maximum vorticity values (during the droplet deformation process) were related to the moment when the droplet was broken and the two new droplets were separated. 


\section{REFERENCES}

Ahmadi, F., K. Samlali, P. Q. N. Vo and S. C. C. Shih (2019). An integrated droplet-digital microfluidic system for on-demand droplet creation, mixing, incubation, and sorting. Lab on a Chip 19, 524-535.

Amani, E., A. Ahmadpour and M. Tohidi (2019). A numerical study of the rise of a Taylor bubble through a sudden/gradual expansion in Newtonian and shear-thinning liquids. International Journal of Mechanical Sciences.

Bedram, A., A. E. Darabi, A. Moosavi and S. Kazemzade (2015a). Numerical Investigation of an Efficient Method (T-Junction With Valve) for Producing Unequal-Sized Droplets in Micro- and Nano-Fluidic Systems. Journal of Fluids Engineering 137, 031202.

Bedram, A. and A. Moosavi (2011). Droplet breakup in an asymmetric microfluidic T junction. Eur. Phys. J. E 34, 78.

Bedram, A. and A. Moosavi (2013). Breakup of Droplets in Micro and Nanofluidic TJunctions. Journal of Applied Fluid Mechanics 6(1), 81-86.

Bedram, A., A. Moosavi and S. Kazemzade Hannani (2015b). Analytical relations for long-droplet breakup in asymmetric T junctions. Physical Review E 91, 053012.

Bretherton, F. P. (1961). The motion of long bubbles in tubes. J.Fluid Mech. 166, 10.

Chiarello, E., A. Gupta, G. Mistura, M. Sbragaglia and M. Pierno (2017). Droplet breakup driven by shear thinning solutions in a microfluidic $\mathrm{T}$ junction. Phys. Rev. Fluids 2, 123602.

Cui, W., G. Yesiloz, and C.L. Ren, (2020). Numerical Analysis on Droplet Mixing Induced by Microwave Heating: Decoupling of Influencing Physical Properties. Chemical Engineering Science.

Dai, C., S. Fang, Y. Wu, X. Wu, M. Zhao, C. Zou, H. Li, H. Zhou and K. Zhang (2017). Experimental study of bubble breakup process in non-Newtonian fluid in 3-D pore-throat microchannels. Colloids and Surfaces A.

Deshpande, S. and C. Dekker (2018). On-chip microfluidic production of cell-sized liposomes. Nature protocols 13, 5 .

Feng, J. Q. (2017). A Computational Study of HighSpeed Microdroplet Impact onto a Smooth Solid Surface. Journal of Applied Fluid Mechanics 10, 1.

Fu, T., Y. Ma and H. Z. Li (2014). Hydrodynamic Feedback on Bubble Breakup at a T-junction Within an Asymmetric Loop. AIChE Journal 60,5 .

$\mathrm{Hu}, \mathrm{X}$. and T. Cubaud (2018).Viscous Wave Breaking and Ligament Formation in
Microfluidic Systems. Physical Review Letters 121,044502 .

Jejurkar, S. Y., G. Yadav and D. P. Mishra (2017). Visualizations of sheet breakup of nonNewtonian gels loaded with nanoparticles. International Journal of Multiphase Flow.

Khoo, B. L., G. Grenci, Y. B. Lim, S. C. Lee, J. Han and C. T. Lim (2018). Expansion of patientderived circulating tumor cells from liquid biopsies using a CTC microfluidic culture device. Nature protocols 13, 34-58.

KianiMoqadam, A., A. Bedram and M. H. Hamedi (2018). A Novel Method (T-Junction with a Tilted Slat) for Controlling Breakup Volume Ratio of Droplets in Micro and Nanofluidic TJunctions. Journal of Applied Fluid Mechanics 11(1), 1255-1265.

Leshansky, A. M. and L. M. Pismen (2009). Breakup of drops in a microfluidic T-junction. Physics of Fluids 21, 023303.

Link, D. R., S. L. Anna, D. A. Weitz and H. A. Stone (2004). Geometrically Mediated Breakup of Drops in Microfluidic Devices. Phys. Rev. Lett.92, 054503.

Liu, Y., J. Yue, S. Zhao, C. Yao and G. Chen (2018) Bubble splitting under gas-liquid-liquid threephase flow in a double T-junction microchannel. AIChE Journal 64, 1.

Maio, L. D. and F. Dunlop (2018). Sessile Drop on Oscillating Incline. Journal of Applied Fluid Mechanics 11, 6.

Mondal, B. and D. Chatterjee (2016). Numerical Investigation of the Water Droplet Transport in a PEM Fuel Cell with Serpentine Flow Channel. Journal of Applied Fluid Mechanics 9, 3 .

Mora, A. E. M., A. L. F. L. Silva and S. M. M. L. Silva (2018). Numerical study of the dynamics of a droplet in a T-junction microchannel using OpenFOAM. Chemical Engineering Science.

Muzychka, Y. S. and J. Edge (2008). Laminar nonNewtonian Fluid Flow in Noncircular Ducts and Microchannels. Journal of Fluids Engineering 130, 111201.

Rostami, B. and G. L. Morini (2020). Generation of Newtonian droplets in Newtonian and nonNewtonian carrier flows in micro T-junctions under opposed-flow configuration. Journal of Non-Newtonian Fluid Mechanics 281, 104297.

Rostami, B. and G. L. Morini (2018). Generation of Newtonian and non-Newtonian droplets in silicone oil flow by means of a micro crossjunction. International Journal of Multiphase Flow.

Rahman, M. M., W. Lee, A. Iyer and S. J. Williams (2019). Viscous resistance in drop coalescence. Phys. Fluids 31, 012104. 
M. J. Kiani and A. Bedram / JAFM, Vol. 15, No. 2, pp. 491-503, 2022.

Ruggeri, F. S., J. Charmet, T. Kartanas, Q. Peter, S. Chia, J. Habchi, C. M. Dobson, M. Vendruscolo and T. P. J. Knowles (2018). Microfluidic deposition for resolving single molecule protein architecture and heterogeneity. Nature Communications 9, 3890 .

Sontti, S. G. and A. Atta (2017). CFD analysis of microfluidic droplet formation in nonNewtonian liquid. Chemical Engineering Journal.

Wang, X., C. Zhu, T. Fu, T. Qiu and Y. Ma (2017). Critical condition for bubble breakup in a microfluidic flow-focusing junction. Chemical Engineering Science 164, 178-187.

Wang, X., C. Zhu, T. Fu, and Y. Ma (2014). Bubble breakup with permanent obstruction in an asymmetric microfluidic T-junction. AIChE Journal 61, Issue 3, 1081-1091.

White, F. M., (2011). Fluid mechanics. $7^{\text {th }}$ edition. Mcgraw-Hill. (Eq. 4.32).
Xiong, Q., C. Y. Lim, J. Ren, J. Zhou, K. Pu, M. B. Chan-Park, H. Mao, Y. C. Lam and H. Duan, (2018). Magnetic nanochain integrated microfluidic biochips. Nature Communications 9, 1743.

Yang, S. H., J. Parka, J. R. Youn and Y. S. Song (2018). Programmable microfluidic logic device fabricated with shape memory polymer. Lab on a Chip.

Yadavali, S., H. H. Jeong, D. Lee and D. Issadore (2018). Silicon and glass very large scale microfluidic droplet integration for tera scale generation of polymer microparticles. Nature Communications 9, 1222.

Zhao, M., M. Cao, Y. Hu, Q. Yang, Y. Wu, Z. Chu, $\mathrm{X}$. $\mathrm{Hu}$ and C. Dai (2020). Investigating breakup behaviors of the non-Newtonian fluid: A case study of oil droplet using 3-D pore throat structured microchannels. Colloids and Surfaces A: Physicochemical and Engineering Aspects 587, 124330. 